\title{
TITLE:
}

\section{The evolution of harmonic mappings with free boundaries}

\section{AUTHOR(S):}

Struwe, Michael

\section{CITATION:}

Struwe, Michael. The evolution of harmonic mappings with free boundaries. 数理解析研 究所講究録 1991, 770: 148-160

\section{ISSUE DATE:}

1991-11

URL:

http://hdl.handle.net/2433/82358

RIGHT: 


\title{
The evolution of harmonic mappings with free boundaries
}

\author{
Michael Struwe \\ Mathematik, ETH-Zentrum, CH-8092 Zürich
}

\begin{abstract}
We establish the existence of a global, partially regular weak solution to the evolution problem for harmonic maps with free boundaries on a suitable support hypersurface.
\end{abstract}

1. Let $(M, g)$ be a $m$-dimensional manifold with boundary $\partial M$ and let $N$ be a compact $\ell$-dimensional manifold, which for convenience we may regard as isometrically embedded in some Euclidean space $\mathbb{I}^{n}$. Also let $\Sigma$ be a $k$-dimensional sub-manifold of $\mathbb{R}^{n}, S=\Sigma \cap N$. Finally, let $u_{0}=\left(u_{0}^{1}, \ldots, u_{0}^{n}\right): M \rightarrow N$ with $u_{0}(\partial M) \subset S$ be given.

We study the existence of harmonic maps $u: M \rightarrow N \hookrightarrow \mathrm{R}^{n}$ solving the free boundary problem

$$
\begin{gathered}
-\Delta u=\Gamma(u)(\nabla u, \nabla u) \perp T_{u}(N), \\
u(\partial M) \subset S, \\
\frac{\partial}{\partial n} u \perp T_{u} S \text { on } \partial M,
\end{gathered}
$$

where $n$ denotes a unit normal vector field along $\partial M, \Delta=\Delta_{M}$ is the LaplaceBeltrami operator on $M$, and $\Gamma$ denotes a bilinear form related to the second fundamental form of the embedding $N \hookrightarrow \mathrm{R}^{n}$. Finally, $T_{p} N$ denotes the tangent space (in $\mathbb{R}^{n}$ ) of $N$ at $p$, and $\perp$ means orthogonal (in $\mathbb{R}^{n}$ ). That is, we look for critical points of the energy

$$
E(u)=\frac{1}{2} \int_{M}|\nabla u|^{2} d M
$$

on the space of maps

$$
H_{S}^{1,2}(M ; N)=\left\{u \in H^{1,2}\left(M ; \mathbb{R}^{n}\right) ; u(M) \subset N, u(\partial M) \subset S\right\} .
$$

Here, $H^{1,2}\left(M ; \mathbb{P}^{n}\right)$ is the Sobolev space of $L^{2}$-maps $u: M \rightarrow \mathbb{R}^{n}$ with $\nabla u \in L^{2}$; the norm $|\nabla u|^{2}$ is computed in the metric on $M$. 
As in [13] for a related problem, we approach (1.1)-(1.3) by means of the evolution problem

$$
\begin{gathered}
u_{t}-\Delta u=\Gamma(u)(\nabla u, \nabla u) \text { on } M \times[0, \infty[, \\
u(x, t) \subset S, \text { for } x \in \partial M, t \geq 0, \\
\frac{\partial}{\partial n} u(x, t) \perp T_{u(x, t)} S, \text { for } x \in \partial M, t>0 \\
u(\cdot, 0)=u_{0} \text { on } M .
\end{gathered}
$$

If $m=2$ this strategy has been sucessfully implemented by $\mathrm{Ma} \mathrm{Li}$ [10]. See also Dierkes-Hildebrandt-Wohlrab [5] and Hildebrandt-Nitsche [7] for further material on the two-dimensional case. Here we confront the higher dimensional case $m \geq 3$. Assume all data are smooth. For simplicity, we consider only the case

$$
M=B=B_{1}(0)=\left\{x \in \mathbb{R}^{m} ;|x|<1\right\} .
$$

Moreover, we make the following assumption about $\Sigma$, the global "extension" of $S$ to the ambient Euclidean space:

There exists a ball $U \subset \mathbb{R}^{n}$ containing $N$, whose boundary $\partial U$ intersects $\Sigma$ orthogonally in the sense that the normal $\nu_{U}$ to $\partial U$ at a point $p \in \Sigma$ lies in $T_{p} \Sigma$.

In addition assume that the nearest neighbor projection $\pi_{\Sigma}: U \rightarrow \Sigma \cap U$ is welldefined and smooth in $U$, and

$$
\left|D^{2} \pi_{\Sigma}\right| \cdot \operatorname{diam}(U)<1 / 2
$$

Let $R_{\Sigma}(p)=2 \pi_{\Sigma}(p)-p$ be the reflection of a point $p \in U$ in $\Sigma$. Also we suppose $\Sigma$ is oriented by a smooth normal frame $\nu=\left(\nu_{1}, \ldots, \nu_{n-k}\right)$. An example of a configuration $(N, \Sigma)$ satisfying (1.9-10) is $N=S^{n-1} \subset \mathbb{R}^{n}, \Sigma=\mathbb{R}^{k} \times\{0\}, k \leq n-1$, or a perturbation of $\mathbb{R}^{k} \times\{0\}$ by a diffeomorphism $\Phi=i d+\varepsilon \tau$, with a smooth map 
$\tau: \mathbb{R}^{n} \rightarrow \mathbb{R}^{n}$ having compact support, and $|\varepsilon|<\varepsilon_{0}=\varepsilon_{0}(\tau)$. Then we obtain the following result reminiscent of the results in [2] for the evolution of harmonic maps on closed domains, that is, with $\partial M=\emptyset$.

Theorem 1.1: Suppose $M=B, N, S, u_{0}$ are as above and $S$ satisfies conditions (1.9-10). Then there exists a global weak solution $u$ of problem (1.5-8) satisfying the energy inequality

$$
\int_{0}^{T} \int_{B}\left|u_{t}\right|^{2} d x d t+E(u(T)) \leq E\left(u_{0}\right)
$$

for all $T \geq 0$, and smooth off a singular set of codimension $\geq 2$. As $t \rightarrow \infty$ suitably, $u(t)$ converges weakly in $H^{1,2}(B ; N)$ to a weak solution $u_{\infty}$ of $(1.1-3)$ which is smooth off a set of codimension $\geq 2$.

Remark 1.1: (i) If the range $u(B \times[0, \infty[)$ lies in a convex neighborhood of a point $p$ on $N, u$ is globally smooth and converges uniformly on $\bar{B}$ to a smooth solution $u_{\infty}$ of (1.1-3) homotopic to $u_{0}$.

(ii) Conversely, for instance in the case of a sphere as target manifold, it is known that solutions to (1.5) may develop singularities in finite time, see [4], [1].

(iii) A result like Theorem 1.1 should also hold without the hypotheses (1.9-10) on $S$; however, for a general support manifold $S$ - already in the Euclidean case $N=\mathbb{R}^{n}$ and in contrast to the two-dimensional case - in higher dimensions $m \geq 3$ the problem of boundary regularity for (1.5) poses considerable difficulties and the construction of global, partially regular solutions to (1.5-8) or (2.1), (1.6-8) below is not yet within reach.

(iv) Similar results should hold on a general compact domain with boundary. In fact, much of what follows is true for such general domains and we keep the notation $M$ in that case.

2. Let $U_{\delta}(N)$ be the $\delta$-tubular neighborhood of $N$ in $\mathbf{R}^{n}$. We may choose $\delta>0$ such that $U_{\delta}(N) \subset U$, see (1.9), and such that the nearest neighbor projection $\pi: U_{\delta}(N) \rightarrow N$ is well-defined and smooth in $U_{\delta}(N)$. Let $\chi \in C_{0}^{\infty}(\mathbb{R})$ be a nondecreasing function satisfying $\chi(s)=s$ for $0<s<\frac{\delta^{2}}{2}, \chi(s)=\delta^{2}$ for $s \geq \delta^{2}$. 
Following the approach of [2], we approximate (1.5-8) by the following evolution problem for maps with range in $\mathrm{P}^{n}$ :

$$
u_{t}-\Delta u+K \chi^{\prime}\left(\operatorname{dist}^{2}(u, N)\right) \frac{d}{d u}\left(\frac{\operatorname{dist}^{2}(u, N)}{2}\right)=0
$$

in $M \times[0, \infty[$, with boundary and initial conditions (1.6-8). (2.1) is the evolution equation for the functional

$$
E_{K}(u)=\frac{1}{2} \int_{M}\left[|\nabla u|^{2}+K \chi\left(\operatorname{dist}^{2}(u, N)\right)\right] d M
$$

for maps $u: M \rightarrow \mathbb{R}^{n}$.

Lemma 2.1: Let $u$ be a smooth solution to (2.1), (1.6-8). Then we have

$$
\int_{0}^{T} \int_{M}\left|u_{t}\right|^{2} d M d t+E_{K}(u(T)) \leq E_{K}\left(u_{0}\right)=E\left(u_{0}\right)
$$

for all $T \geq 0$.

Proof: Multiply (2.1) by $u_{t}$ and integrate by parts. The boundary term vanishes on account of (1.6-7).

For the following result hypotheses (1.9-10) on $S$ are essential.

Lemma 2.2: Suppose $u \in C^{1}\left(\bar{M} \times\left[0, T\left[; \mathbb{R}^{n}\right)\right.\right.$ is a smooth solution to (2.1), (1.6-8) on $\bar{M} \times[0, T[$; then $u$ and its first spatial derivatives are uniformly bounded and $u$ extends to a smooth solution of $(2.1),(1.6-8)$ on $\bar{M} \times[0, T]$.

Proof: The interior estimates easily follow from the energy estimate Lemma 2.1 and the interior regularity estimates for the heat equation; see for instance [9]. To obtain the estimates at the boundary we argue as follows. Note that by the maximum principle for the heat equation and (1.6-7), (1.9) the image of $u$ satisfies $u(x, t) \in U$ for all $(x, t)$, and by (1.10) the reflection of $u$ in $\Sigma$ is defined. Thus, in the special case $M=B$, for $x \in \mathbb{R}^{m}, t \geq 0$ we may let

$$
\tilde{u}(x, t)= \begin{cases}u(x, t) & , \text { if }|x|<1 \\ R_{\Sigma}\left(u\left(x /|x|^{2}\right)\right) & , \text { if }|x|>1\end{cases}
$$


Then $\tilde{u}$ is of class $C^{1}$ on $\mathbb{R}^{m} \times[0, T[$ and satisfies

$$
\left|\tilde{u}_{t}+A \tilde{u}\right| \leqq \begin{cases}C K & , \text { if }|x|<1 \\ C K+\Gamma_{\Sigma}(\tilde{u})(\nabla \tilde{u}, \nabla \tilde{u}) & , \text { if }|x|>1\end{cases}
$$

where $A$ is an elliptic operator in divergence form with Lipschitz coefficients, $A=$ $-\Delta$ for $|x|<1$, and where $\Gamma_{\Sigma}$ is a bilinear form related to the second fundamental form of $\Sigma \subset \mathbb{R}^{n}$.

In fact, from

$$
\begin{aligned}
\left(\tilde{u}_{t}+A \tilde{u}\right)\left(\frac{x}{|x|^{2}}\right) & :=\left(2\left(\partial_{t}-\Delta\right) \pi_{\Sigma}(u)-\left(\partial_{t}-\Delta\right) u\right)(x, t)= \\
& =\left(2\left[D \pi_{\Sigma}(u)-i d\right]\left[\left(\partial_{t}-\Delta\right) u\right]-2 D^{2} \pi_{\Sigma}(u)(\nabla u, \nabla u)\right)(x, t)
\end{aligned}
$$

we can read off the precise form of $A$ and $\Gamma_{\Sigma} \cdot(2.3)$ is a parabolic system of the type

$$
u_{t}+A u=f(\cdot, u, \nabla u)
$$

on any ball $B_{\rho}=B_{\rho}(0)$, where

$$
|f(\cdot, u, p)| \leq a|p|^{2}+b
$$

with constants $a, b \in \mathbb{R}$. Moreover, by (1.10), for $\rho>1$ sufficiently close to 1 there holds

$$
a \cdot \sup |u|<\lambda
$$

where $\lambda>0$ denotes the ellipticity constant of the operator $A$ on $B_{\rho}$. By the results of [6] for such systems, $\tilde{u}$ is locally Hölder continuous on $\left.\left.B_{\rho} \times\right] 0, T\right]$. Higher regularity $\left|\nabla^{2} \tilde{u}\right| \in L_{\text {loc }}^{2}\left(B_{\rho} \times[0, T]\right),|\nabla \tilde{u}| \in L_{l o c}^{4}\left(B_{\rho} \times[0, T]\right)$ then follows as in [9]. Finally, by $\left[9\right.$; p. 593f.] we also obtain uniform bounds for $\nabla \tilde{u}$ in $L_{\text {loc }}^{2 p}$ and hence $\tilde{u}_{t}$ and $\nabla^{2} \tilde{u}$ in $L_{l o c}^{p}$ for all $p<\infty$. By the Sobolev embedding theorem [9; Lemma II. 3.3] this then implies the desired bound. 
The a-priori bounds of Lemma 2.2 now yield the following global existence result.

Proposition 2.1: Under the hypotheses of Theorem 1.1, for any $K \in \mathbb{I N}$ there exists a global solution $u=u_{K} \in C^{1}\left(\bar{B} \times\left[0, \infty\left[; \mathrm{R}^{n}\right)\right.\right.$ to $(2.1),(1.6-8)$. The solution $u$ is smooth in $\bar{B} \times[0, \infty[$ and satisfies the energy inequality Lemma 2.1 .

Proof: Local existence follows from a fixed point argument as in [13]. For completeness we sketch the argument. Extend $u_{0}$ to $\mathbb{R}^{m}$ by letting

$$
u_{0}(x)=R_{\Sigma}\left(u\left(\frac{x}{|x|^{2}}\right)\right)
$$

for $x \notin \bar{B}$, and fix $\rho>0, T>0$ sufficiently small. Let

$$
V_{\rho}(T)=\left\{u \in C^{1,1 / 2}\left(\overline{B_{\rho}} \times[0, T] ; \mathbb{R}^{n}\right) ; u(0)=u_{0}\right\}
$$

where $C^{1,1 / 2}(\ldots)$ is the space of functions $u$ which are continuously differentiable in the spatial variable $x$ and uniformly Hölder continuous in time with Hölder exponent $\frac{1}{2}$. A norm is given by the Hölder constant and $\|\nabla u\|_{L^{\infty}}$. - In [9;p.7f.] this space is introduced as $H^{1,1 / 2}$.

For $u \in V_{\rho}(T)$ let $v$ solve

$$
v_{t}+A v= \begin{cases}K \chi^{\prime}\left(\operatorname{dist}^{2}(u, N)\right) \frac{d}{d u}\left(\frac{\operatorname{dist}^{2}(u, N)}{2}\right), & \text { if }|x|<1 \\ K \chi^{\prime}(\ldots) \frac{d}{d u}(\ldots)+\Gamma_{\Sigma}(u)(\nabla u \nabla u), & \text { if }|x|>1\end{cases}
$$

on $B_{\rho} \times[0, T]$ with boundary and initial data $u$. By the interior estimates for the heat equation we can bound $v$ and its first and second derivatives in Hölder norm on $\partial B_{1 / \rho} \times[0, T]$ in terms of the $C^{1,1 / 2}$-norm of $u$ on $B_{\rho} \times[0, T]$ and $u_{0}$. Define new $C^{2}$-Dirichlet data by letting

$$
w(x, t)=R_{\Sigma}\left(v\left(\frac{x}{|x|^{2}}, t\right)\right), x \in \partial B_{\rho}
$$

and let $\bar{u}$ solve (2.5) with initial data $u_{0}$ and boundary data $w$. By (2.4) w and $u_{0}$ are compatible. Moreover, by the linear estimates for the heat equation (see [7; Theorem IV. 9.1] ) the map $F: u \mapsto \bar{u}$ is bounded from $C^{1, \frac{1}{2}}\left(\overline{B_{\rho}} \times[0, T]\right)$ into the space

$$
W_{p}^{2,1}=\left\{u \in L^{p}\left(B_{\rho} \times[0, T]\right) ; u_{t}, \nabla^{2} u \in L^{p}\right\}
$$


for all $p<\infty$, which for $p>m+2$ is compactly embedded into $C^{1, \frac{1}{2}}\left(\overline{B_{\rho}} \times[0, T]\right)$; see [9; Lemma II.3.3]. Finally, if $T>0$ is sufficiently small, $F$ maps a convex $C^{1, \frac{1}{2}}$-neighborhood of the function $u(t) \equiv u_{0}$ to itself. Hence $F$ has a fixed point $u=F(u)$, satisfying (2.5) and the condition

$$
u(x, t)=w(x, t)=R_{\Sigma}\left(v\left(x /|x|^{2}, t\right)\right)
$$

on $\partial B_{\rho} \times[0, T]$. But then also $u_{1}(x, t)=R_{\Sigma}\left(u\left(\frac{x}{|x|^{2}}, t\right)\right)$ is a solution of $(2.5)$ in $\{(x, t) ; 1 / \rho<|x|<\rho\}$ with the same initial and boundary data. It follows that $u=u_{1}$ and thus $u$ satisfies (2.1), (1.6-8). The local solution can be continued globally on account of Lemma 2.2 .

To derive uniform interior estimates independent of $K$ we need the following analogue of the monotonicity formula from [14]. Fix $\left.z_{0}=\left(x_{0}, t_{0}\right) \in \bar{M} \times\right] 0, \infty[$. Let

$$
G(x, t)=\frac{1}{\sqrt{4 \pi|t|}^{m}} \exp \left(-\frac{|x|^{2}}{4|t|}\right)
$$

be the fundamental solution to the heat equation. Then let

$$
\Phi_{z_{0}}(R)=\Phi_{z_{0}}(R ; u, K)=\frac{1}{2} R^{2} \int\left[|\nabla u|^{2}+K \chi\left(\operatorname{dist}^{2}(u, N)\right)\right] G\left(\cdot-z_{0}\right) d x
$$

where we integrate over $B \times\left\{t_{0}-R^{2}\right\}$. On a general domain we would need to localize $\Phi$ in coordinate charts via suitable cut-off functions, as in [2].

Lemma 2.3: There exist constants depending only on $M$ and $N$ such that for all $z_{0}=\left(x_{0}, t_{0}\right)$ and $0 \leq R \leq R_{0} \leq \sqrt{t_{0}}$ there holds

$$
\Phi_{z_{0}}(R) \leq \exp \left(c\left(R_{0}-R\right)\right) \Phi_{z_{0}}(R)+c E\left(u_{0}\right)\left(R_{0}-R\right)
$$

Proof: At interior points this result was obtained in [2; Lemma 4.2]. At the boundary, for simplicity we present the proof only for a half-space $M=\mathbb{R}_{+}^{m}$, where

$$
\mathbb{R}_{+}^{m}=\left\{x=\left(x^{\prime}, x_{m}\right) \in \mathbb{R}^{m} ; x_{m}>0\right\}
$$

and $z_{0}=(0,0)$. (The general case then follows as in [2].) Consider the family of scaled maps

$$
u_{R}(x, t)=u\left(R x, R^{2} t\right)
$$


Note that $u_{R}$ satisfies (2.1) with $R^{2} K$ instead of $K$, and also satisfies (1.6), (1.7). Moreover,

$$
\Phi_{0}(R ; u, K)=\Phi_{0}\left(1 ; u_{R}, R^{2} K\right)
$$

whence (at $R=1$, say)

$$
\begin{aligned}
\frac{d}{d R} \Phi_{0}(R ; u, K)= & \frac{d}{d R} \Phi_{0}\left(1 ; u_{R}, R^{2} K\right) \\
=\int_{S_{+}}\left\{\nabla u \nabla\left(\frac{d}{d R} u_{R}\right)\right. & +K \chi\left(\operatorname{dist}^{2}(u, N)\right) \\
& \left.+K \chi^{\prime}(\ldots) \frac{d}{d u}\left(\frac{\operatorname{dist}^{2}(u, N)}{2}\right) \frac{d}{d R} u_{R}\right\} G d x
\end{aligned}
$$

where $S_{+}=\mathbb{R}_{+}^{m} \times\{-1\}$. Integrating by parts in the first term, on account of (2.1) and the fact that $\nabla G=\frac{x}{2 t} G$, this gives

$$
=\int_{S_{+}} \frac{\left|x \cdot \nabla u+2 t u_{t}\right|^{2}}{2|t|} G d x+\int_{S_{+}} K \chi\left(\operatorname{dist}^{2}(u, N)\right) G d x \geq 0
$$

as desired. Note that by (1.6-7) no boundary terms appear.

Denote by

$$
e_{K}(u)=\frac{1}{2}\left\{|\nabla u|^{2}+K \chi\left(\operatorname{dist}^{2}(\mathrm{u}, \mathrm{N})\right)\right\}
$$

the energy density for the penalized equation. For a point $z_{0}=\left(x_{0}, t_{0}\right) \in \mathbb{R}^{m} \times \mathbb{R}$, $\rho>0$ also denote

$$
P_{\rho}\left(z_{0}\right)=\left\{z=(x, t) ;\left|x-x_{0}\right|<\rho, t_{0}-\rho^{2}<t<t_{0}\right\}
$$

the parabolic cylinder of radius $\rho$ centered at $z_{0}, P_{\rho}=P_{\rho}(0)$ for brevity, and let

$$
\begin{aligned}
& P_{\rho}^{+}\left(z_{0}\right)=P_{\rho}\left(z_{0}\right) \cap\left\{x_{m}>0\right\}, \\
& P_{\rho}^{-}\left(z_{0}\right)=P_{\rho}\left(z_{0}\right) \cap\left\{x_{m}<0\right\},
\end{aligned}
$$

respectively.

Lemma 2.4: There exists a constant $\varepsilon_{0}>0$ depending only on $M$ and $N$ with the following property: If for some $\left.z_{0}=\left(x_{0}, t_{0}\right) \in \bar{M} \times\right] 0, \infty\left[\right.$ and $R<\varepsilon_{0}$ the inequality

$$
\Phi_{z_{0}}\left(R ; u_{K}, K\right)<\varepsilon_{0}
$$


is satisfied, then

$$
\sup _{P_{\delta R}\left(z_{0}\right)} e_{K}\left(u_{K}\right) \leq c(\delta R)^{-2}
$$

with constants $c$ depending only on $M$ and $N$ and $\delta>0$ possibly depending also on $E\left(u_{0}\right)$ and $\min \{R, 1\}$.

Proof: The proof for interior points $x_{0} \in M$ is the same as that of Lemmas 2.4, 4.4 of [2]. We sketch the modifications at a boundary point $x_{0}$. Again assume for simplicity that $M=\mathbb{R}_{+}^{m}$ and shift $z_{0}$ to 0 . By reflection we may extend $u$ to a solution $\tilde{u}$ of

$$
\tilde{u}_{t}-\Delta \tilde{u}= \begin{cases}K \chi^{\prime}\left(\operatorname{dist}^{2}(\tilde{u}, N)\right) \frac{d}{d u}\left(\frac{\operatorname{dist}^{2}(\tilde{u}, N)}{2}\right), & \text { if } x_{m}>0 \\ K \chi^{\prime}(\ldots) \frac{d}{d u}(\ldots)+\Gamma_{\Sigma}(\tilde{u})(\nabla \tilde{u}, \nabla \tilde{u}), & \text { if } x_{m}<0\end{cases}
$$

on a full neighborhood of $x_{0}$. Scaling as in [2; p. 92], we obtain a solution $v$ of problem (2.6) for some $\tilde{K}=\frac{K}{e_{0}}$ on $P_{1}$, satisfying

$$
e_{\tilde{K}}(v) \leq 4
$$

and

$$
e_{\tilde{K}}(v)(0)=1
$$

Moreover, we have the differential inequality

$$
\left(\partial_{t}-\Delta\right) e_{K}(v)+\left|\nabla^{2} v\right|^{2} \leq C e_{K}(v)
$$

separately in $P_{1}^{+}$and $P_{1}^{-}$. (The proof of this Bochner-type estimate can be conveyed very easily from $\left[2 ;\right.$ p. 90].) Let us for brevity write $e_{\tilde{K}}(v)=e(v)$ in the sequel. Our aim is to extend (2.7) to $P_{1}$.

Due to the structure of $(2.6), \Delta e(v)$ may have a singular component on the hypersurface $\left\{x_{m}=0\right\}$ - in our old coordinates. As in [13], we may control this component in the following way.

Given $\varphi \in C_{0}^{\infty}(B),-1<t<0$, we have

$$
-\int \Delta e(v) \varphi^{2} d x=\int_{\left\{x_{m}=0\right\}}\left[\partial_{x_{m}} e(v)\right]_{-}^{+} \varphi^{2} d x^{\prime}+2 \int \nabla e(v) \nabla \varphi \varphi d x
$$

where $\int \ldots$ denotes integration over $B \times\{t\}$, and where we denote

$$
\left[f\left(x^{\prime}, 0\right)\right]_{-}^{+}=\lim _{x_{m} \searrow 0} f\left(x^{\prime}, x_{m}\right)-\lim _{x_{m} \nearrow_{0}} f\left(x^{\prime}, x_{m}\right)
$$


for any function $f$.

To estimate the boundary integral we decompose

$$
\begin{aligned}
{\left[\partial_{x_{m}} e(v)\right]_{-}^{+} } & =\frac{1}{2}\left[\partial_{x_{m}}\left(|\nabla v|^{2}\right)\right]_{-}^{+}+\frac{\tilde{K}}{2}\left[\partial_{x_{m}} \chi\left(\operatorname{dist}^{2}(\mathrm{v}, \mathrm{N})\right)\right]_{-}^{+} \\
& =\frac{1}{2}\left[\partial_{x_{m}}\left(|\nabla v|^{2}\right)\right]_{-}^{+} \\
& =\left[\partial_{x_{m}}^{2} v \partial_{x_{m}} v\right]_{-}^{+}+\left[\partial_{x_{m}}\left(\nabla_{x^{\prime}} v\right) \nabla_{x^{\prime}} v\right]_{-}^{+} \\
& =\left[\Delta v \partial_{x_{m}} v\right]_{-}^{+}-2\left[\Delta_{x^{\prime}} v \partial_{x_{m}} v\right]_{-}^{+}+\left[\nabla_{x^{\prime}} \cdot\left(\partial_{x_{m}} v \nabla_{x^{\prime}} v\right)\right]_{-}^{+}
\end{aligned}
$$

But by (1.6), (1.7)

$$
\partial_{x_{m}} v \nabla_{x^{\prime}} v=0
$$

Hence, and on account of (2.6), (1.6), we have

$$
\left[\partial_{x_{m}} e(v)\right]_{-}^{+}=\left\langle\Gamma_{\Sigma}(v)(\nabla v, \nabla v), \partial_{x_{m}} v\right\rangle-2\left[\Delta_{x^{\prime}} v \partial_{x_{m}} v\right]_{-}^{+}
$$

where for clarity we now denote $\langle\cdot, \cdot\rangle$ the scalar product in $\mathbb{R}^{n}$. Using the normal frame $\nu=\left(\nu_{1}, \ldots, \nu_{n-k}\right)$ for $\Sigma$, the last term by (1.7) may be more conveniently written

$$
\begin{aligned}
\Delta_{x^{\prime}} v \partial_{x_{m}} v & =\sum_{j}\left\langle\Delta_{x^{\prime}} v, \nu_{j}(v)\right\rangle\left\langle\nu_{j}(v), \partial_{x_{m}} v\right\rangle \\
& =-\sum_{j}\left\langle\nabla_{x^{\prime}} v, \nabla_{x^{\prime}}\left(\nu_{j}(v)\right)\right\rangle\left\langle\nu_{j}(v), \partial_{x_{m}} v\right\rangle .
\end{aligned}
$$

Smoothly extend $\nu_{j}$ to $\mathbb{R}^{n}$. Then by the divergence theorem

$$
\begin{aligned}
& \underset{\left\{x_{m}=0\right\}}{\int\left[\partial_{x_{m}} e(v)\right]_{-}^{+} \varphi^{2} d x^{\prime}=} \int_{P_{1}^{-}} \operatorname{div}\left(\left\langle\Gamma_{\Sigma}(v)(\nabla v, \nabla v), \nabla v\right\rangle \varphi^{2}\right) d x \\
& \mp \sum_{j} \int_{P_{1}^{ \pm}} \operatorname{div}\left(\left\langle\nabla_{x^{\prime}} v, \nabla_{x^{\prime}}\left(\nu_{j}(v)\right)\right\rangle\left\langle\nu_{j}(v), \nabla v\right\rangle \varphi^{2}\right) d x \\
& \leq C \int_{P_{1}}\left(\left|\nabla^{2} v\right||\nabla v|^{2}+|\nabla v|^{4}\right) \varphi^{2} d x+C \int_{P_{1}}|\nabla v|^{3}|\nabla \varphi||\varphi| d x \\
& \leq \varepsilon \int_{P_{1}}\left|\nabla^{2} v\right|^{2} \varphi^{2} d x+C(\varepsilon) \int|\nabla v|^{4} \varphi^{2} d x \\
&+C(\varepsilon) \int_{P_{1}}|\nabla v|^{2}|\nabla \varphi|^{2} d x
\end{aligned}
$$


and - choosing $\varepsilon>0$ sufficiently small - it follows that the inequality (2.7) - up to a factor - holds on $P_{1}$ in the distribution sense. But then the remainder of the proof of [2] applies also in this case.

As in [2], we may now pass to the limit $K \rightarrow \infty$. Let $u_{K}$ be a sequence of smooth solutions to (2.1), (1.6-8). We may assume that $u_{K}$ converges weakly to $u$ in the sense

$$
\begin{aligned}
\nabla u_{K} & \rightarrow \nabla u \quad \text { weakly }-* \text { in } L^{\infty}\left(\left[0, \infty\left[; L^{2}(M)\right)\right.\right. \\
\frac{\partial}{\partial t} u_{K} & -\frac{\partial}{\partial t} u \quad \text { weakly in } L^{2}(M \times[0, \infty[) \\
u_{K} & \rightarrow u \text { strongly in } L_{l o c}^{2}(M \times[0, \infty[)
\end{aligned}
$$

and almost everywhere, where $u: \bar{M} \times[0, \infty[\rightarrow N$.

Proposition 2.2: The limit $u$ weakly solves problem (1.5-8). Moreover, $u$ is smooth and solves (1.5) classically on a dense relatively open set $Q_{0} \subset \bar{M} \times[0, \infty[$ whose complement $Q^{\prime}$ has locally finite $(m-2)$-dimensional Hausdorff measure on each time slice $\bar{M} \times\{t=$ const. $\}$. Moreover, $u$ satisfies the energy inequality

$$
\int_{0}^{T} \int_{M}\left|u_{t}\right|^{2} d M d t+E(u(T)) \leq E\left(u_{0}\right)
$$

for all $T>0$. Finally, as $t \rightarrow \infty$ suitably, a sequence $u(\cdot, t)$ converges weakly in $H^{1,2}(M ; N)$ to a solution $u_{\infty}$ of (1.1-3) with $E\left(u_{\infty}\right) \leq E\left(u_{0}\right)$ and smooth away from a closed set $Q^{\prime \prime}$ of finite $(m-2)$-dimensional Hausdorff measure.

Proof: All proofs except (1.3), (1.7) are identical with those of [ 12; Theorem 6.1], resp. [2; Theorem 1.5] in the case of harmonic maps on domains without boundary. See [3] for an estimate of $H^{m-2}\left(Q^{\prime} \cap\{t=\right.$ const. $\left.\}\right)$. To see (1.3), (1.7) in the case of a half-plane we extend $u_{K}$ by reflection to solutions $\tilde{u}_{K}$ of equations (2.6), converging weakly locally to a function $\tilde{u}$. On $Q_{0}$, as in [2; p. 94], we have $C^{1}$-convergence $u_{K} \rightarrow u$, and (1.7) holds on $Q_{0}$. Moreover, there holds $K \cdot \operatorname{dist}(u, N) \rightarrow \lambda$ weakly in $L_{l o c}^{2}\left(Q_{0}\right)$, whence

$$
\tilde{u}_{t}-\Delta \tilde{u} \in L_{l o c}^{2}\left(Q_{0}\right)
$$


Now let $\varphi$ be an arbitrary testing function and let $\eta \in H^{1, \infty}, 0 \leq \eta \leq 1, \eta=0$ in a neighborhood of $Q^{\prime}$, as in [2; p. 95]. Multiplying (2.7) by $\varphi \eta$, we obtain that

$$
\int_{0}^{\infty} \int_{\mathbb{R}^{m}}\left(\tilde{u}_{t}-\Delta \tilde{u}\right) \varphi \eta d x d t=\int_{0}^{\infty} \int_{\mathbb{R}^{m}}\left\{\tilde{u}_{t} \varphi+\nabla \tilde{u} \nabla \varphi\right\} \eta d x d t+F
$$

where

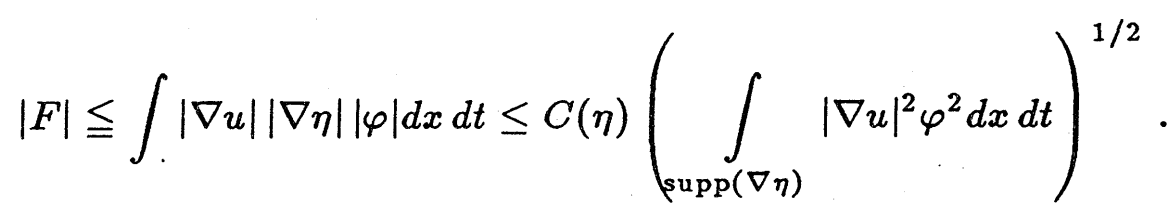

As in [2] we may choose a sequence of maps $\eta$ as above with a uniform constant $C(\eta)=C$ such that $\eta \rightarrow 1$ almost everywhere and $(\operatorname{supp}(\nabla \eta)) \rightarrow 0$ in measure. By absolute continuity of the Lebesgue integral, thus $F \rightarrow 0$, and (1.7) also holds in the distribution sense. The proof of (1.3) is similar.

Theorem 1.1 is an immediate consequence of Proposition 2.2. Remark 1.1 follows by adapting the argument of [8] to our problem. Since this technique is by now well-known we may omit the details.

\section{REFERENCES}

[1] Chen, Y. - Ding, W.: "Blow-up and global existence for heat, flows of harmonic maps", Invent. Math. 99 (1990), 567-578.

[2] Chen, Y. - Struwe, M.: "Existence and partial regularity results for the heat flow for harmonic maps", Math. Z. 201 (1989), 83-103.

[3] Cheng, X:, "Estimate of singular set of the evolution problem for harmonic maps", preprint (1990).

[4] Coron, J.-M. - Ghidaglia, J.-M.: "Explosion en temps fini pour le flot des applications harmoniques", C.R. Acad. Sci. Paris 308, Ser. I (1989), 339-344.

[5] Dierkes, U. - Hildebrandt, S. - Wohlrab, O.: (to appear).

[6] Giaquinta, M. - Struwe, M.: "An optimal regularity result for a class of quasilinear parabolic systems", manusc. math. 36 (1981), 223-239. 
[7] Hildebrandt, S. - Nitsche, J.C.C.: "Minimal surfaces with free boundaries", Acta Math. 143 (1979), 251-272.

[8] Jost, J.: "Ein Existenzbeweis für harmonische Abbildungen, die ein Dirichletproblem lösen, mittels der Methode des Wärmeflusses", manusc. math. 34 (1981), 17-25.

[9] Ladyženskaja, O.A. - Solonnikov, V.A. - Ural'ceva, N.N.: "Linear and quasilinear equations of parabolic type", Amer. Math. Soc. Transl. Math. Monographs 23, Providence (1968).

[10] Ma Li: "Harmonic map heat flow with free boundary", preprint, Trieste (1990).

[11] Schoen, R.M.: "Analytic aspects of the harmonic map problem", Seminar on Nonlinear P.D.E. (Chern, ed.), Springer, Berlin (1984).

[12] Schoen, R.M. - Uhlenbeck, K.: "A regularity theory for harmonic maps", J. Diff. Geom. 17 (1982), 307-335.

[13] Struwe, M.: "The existence of surfaces of constant mean curvature with free boundaries", Acta Math. 160 (1988), 19-64.

[14] M. Struwe, "On the evolution of harmonic maps in higher dimensions", J. Diff. Geom. 28 (1988), 485-502. 